Meta

Journal des traducteurs

Translators' Journal

\title{
Ivre/drunk : étude comparative d'une série synonymique en français et en anglais
}

\section{Claude Romney}

Volume 22, numéro 2, juin 1977

URI : https://id.erudit.org/iderudit/003980ar

DOI : https://doi.org/10.7202/003980ar

Aller au sommaire du numéro

Éditeur(s)

Les Presses de l'Université de Montréal

ISSN

0026-0452 (imprimé)

1492-1421 (numérique)

Découvrir la revue

Citer cet article

Romney, C. (1977). Ivre/drunk : étude comparative d'une série synonymique en

français et en anglais. Meta, 22(2), 132-137. https://doi.org/10.7202/003980ar d'utilisation que vous pouvez consulter en ligne.

https://apropos.erudit.org/fr/usagers/politique-dutilisation/ 


\section{IVRE/DRUNK : ÉTUDE COMPARATIVE DYUNE SERIE SYNONYMIQUE EN FRANÇAIS ET EN ANGLAIS}

Selon Harold Wentworth et Stuart Berg Flexner, auteurs du Dictionary of American Slang ${ }^{1}$, le domaine de l'ivresse est le plus riche du royaume de l'argot. Nous avons nous-même relevé plus de 360 synonymes de drunk dans différents dictionnaires anglais et américains, dont la plupart appartiennent à la langue populaire et argotique. Le français, langue parlée par le peuple du monde qui a la réputation d'être le plus porté sur la boisson, n'en compte qu'environ le tiers. Toutefois, en anglo-américain comme en français, un sondage auprès d'informa-

1. Harold Wentworth et Stuart Berg Flexner, Dictionary of American Slang, New York, Thomas Y. Crowell Co., 1960, p. 652 . 
teurs pouvant être considérés comme avertis a révélé qu'ils ne connaissaient qu'environ le quart des termes dénombrés.

À l'époque de la prohibition aux États-Unis, l'écrivain et critique iittéraire Edmund Wilson avait déjà dressé une liste d'une centaine de synonymes de drunk d'usage courant ${ }^{2}$, et on pourrait d'ailleurs étudier le vocabulaire de l'alcoolisme dans l'œuvre qu'Alfred Kazin nomme les «American literary drunks», mais tel n'est pas ici notre propos. D'un autre côté, il est évident que les traducteurs de Scott Fitzgerald, Hemingway et Thornton Wilder, pour n'en citer que trois, ont dû au moins effleurer le problème des équivalences et donc comparer, dans une certaine mesure, les systèmes anglo-américain et français.

Les dictionnaires bilingues ne sont à cet égard pour le traducteur, ou le curieux, que d'un piètre secours. Ainsi le Harrap's French-English Dictionary of Slang and Colloquialisms ${ }^{3}$ traduit la plupart des synonymes d' ' ivre » par drunk ou dead-drunk, en donnant en fin d'ouvrage une liste de termes populaires ou argotiques parmi lesquels l'utilisateur devra lui-même choisir.

D'un point de vue théorique, les listes de synonymes dans leur totalité présentent un intérêt pour l'étude comparative des deux langues. On pourra ainsi les examiner en tenant compte du niveau de langue, du degré d'ivresse, de la boisson absorbée, des connotations suggérées par le terme, de l'image ou de la comparaison qu'il renferme, et aussi de l'usage du locuteur comme du lecteur. En effet, il tombe sous le sens que l'usage nord-américain diffère de l'usage britannique ou australien et que les termes utilisés en français canadien ne sont pas toujours ceux qu'on emploie en France.

\section{NIVEAU DE LANGUE}

\section{a) Langue soutenue}

On peut assez facilement mettre en parallèle les termes de la langue soutenue qui sont bien définis, par exemple, en américain par le dictionnaire Webster ${ }^{4}$ et en français par le Dictionnaire des synonymes de Bailly ${ }^{5}$. "Ivre » semble ainsi correspondre à drunk, ces deux termes étant les plus généraux. "Éméché » traduirait intoxicated qui dénote un état d'ivresse moins avancé que le couple précédent. Les difficultés commencent cependant avec inebriated qui suggère l'idée d'ivresse bruyante et pour lequel le Harrap's ${ }^{6}$ donne simplement "pris de boisson », alors qu'en français, l'expression de la même famille, «en état d'ébriété », appartient surtout à la langue administrative. Du français vers l'anglais, il est délicat de traduire «soûl », adjectif plus familier qu'« ivre », et plus péjoratif, pour lequel drunk est quelque peu trop neutre et tipsy donne une indication trop précise sur le

2. Cité par Alfred Kazin, "The Giant Killer: Drink and the American Writer », Commentary, mars 1976 , p. 44-50.

3. Joseph Marks, Harrap's French-English Dictionary of Slang and Colloquialisms, Londres, George G. Harrap and Co., 1970

4. Webster's Third New International Dictionary of the English Language, Springfield (Mass.), G. \& C. Merriam Co., 16 éd., 1971.

5. René Bailly, Dictionnaire des synonymes, Paris, Larousse, 1946.

6. J.E. Mansion, Harrap's Standard French and English Dictionary, 2 vol., Londres, G. Harrap and Co., 1970, (1re éd., 1934). 
degré d'ivresse. «Gris » et «pompette » sont légèrement familiers et correspondraient à tipsy et tight.

\section{b) Langue populaire et argotique}

À la limite de la langue familière se situent quelques euphémismes, comme under the influence ou «dans un état voisin », mais c'est à la langue proprement populaire et argotique qu'appartient la quasi-totalité des termes dénombrés. Pour établir des correspondances, il faudra tenir compte des critères cités plus haut et que nous allons examiner à présent.

\section{DEGRÉ D'IVRESSE}

J.C. Hotten, en 1887, décrivait en termes bien victoriens la progression croissante du degré d'ivresse qu'il pouvait constater dans la série synonymique de drunk: de mild intoxication, en passant par a higher or more intense state of beastliness, au climax of fuddlement? . Une hiérarchie pourrait également être proposée en français, et on pourrait l'indiquer, dans les deux langues, à l'aide, par exemple, d'un, deux ou trois signes typographiques fr. : éméché ${ }^{+}, \mathrm{paf}^{++}$, noir $^{+++}$, angl. : primed ${ }^{+}$, soused ${ }^{++}$, pickled ${ }^{+++}$, etc. Les correspondances seraient plus faciles à établir si les dictionnaires comportaient des indications de ce genre.

\section{BOISSON ABSORBÉE}

Chacun sait qu'en France, la boisson nationale est le vin, sauf dans le nord et l'est du pays où l'on boit de la bière. En Amérique du Nord et en Grande-Bretagne, les habitudes dépendent peut-être autant de la classe sociale que du milieu géographique des buveurs. Des adjectifs comme beery et winey, ce dernier d'ailleurs d'usage peu courant, indiquent immédiatement la nature de la boisson. L'expression savoureuse "être dans les vignes du Seigneur», dont la bonhomie semble être légèrement passée de mode, n'aura pas d'équivalent exact en anglais car ces vignes ne pourraient se trouver ailleurs qu'en France.

\section{IMAGES ET COMPARAISONS}

La classification des images, dont bon nombre sont des euphémismes, proposée par Wentworth et Flexner ${ }^{8}$, peut s'appliquer aussi bien au système français qu'à l'anglo-américain. Les catégories d'images contenues dans la plupart des synonymes d'ivre/drunk sont les suivantes, et nous les illustrons ici à l'aide de quelques exemples :

1) « of being high » angl. : elevated, flying high

fr. : lancé, monté, parti

2) « happy, content, bright or conspicuous » angl. : happy, merry, jolly, lit fr. : gai, allumé

7. J.C. Hotten, The Slang Dictionary, Londres, Chatto and Windus, 1887, (réimpression, Totowa, N.J., Rowan and Littlefield, 1972), p. 69.

8. Op. cit., p. 652. 
3) "unconscious or dead" angl. : dead to the world, paralysed, blotto fr. : machabé, dans le brouillard, dans le coma

4) «staggering, especially as a sailor on rough seas angl. : to have a full cargo aboard, to have three (four) sheets in the wind, half-seas-over fr. : avoir du vent dans les voiles

5) « mugged, battered, plastered » angl. : knocked up fr. : culbuté

6) «bottled or caoked 》 angl. : canned, pickled fr. : cuit

7) «a few nonsense words 》 angl. : topsy-boozy, woozy fr. : brindezingue, murdingué, rondibé

Les procédés de formation sont ici soit la suffixation parasitaire, soit l'emploi de l'onomatopée ou d'« une certaine correspondance entre la forme et le sens », procédés qu'utilise volontiers, selon Pierre Guiraud ${ }^{\circ}$, la langue populaire.

À ces sept catégories, on pourrait en ajouter au moins quatre :

8) «l'idée de plénitude» angl. : full fr. : plein, rond, arrondi, farci, gavé

9) «l'idée d'humidité, sous forme liquide ou solide» angl. : wet, lubricated, oiled fr. : humecté, mouillé, gelé, givre

10) «l'idée de couleur» angl. : blued, pinko, rosy fr. : gris, noir

À noter la différence de tons utilisés par les deux langues, le français reflétant l'état intérieur du buveur, l'anglais plutôt l'aspect physique.

11) une référence à un nom de lieu, réel ou fictif, en particulier dans des expressions pittoresques comme en anglais in Liquor Pond Street et en français atteint de la fièvre de Bercy et de la paroisse de Saint-Jean-le-Rond.

Certaines images sont plus fréquentes dans une langue que dans l'autre. Ainsi l'anglais britannique utilise davantage d'expressions empruntées au vocabulaire de la marine : voir catégorie 4 ci-dessus.

9. Pierre Guiraud, 'Argot, Paris, Presses Universitaires de France, 6e édition, 1973, p. 70 et 86, (1 ${ }^{\text {re }}$ édition, 1956). 
Les comparaisons drunk as/soûl comme sont également bien plus nombreuses en anglais où l'on en trouve au mơins une vingtaine, qu'en français qui n'en compte qu'à peine la moitié. Il semble d'ailleurs que l'ivresse ne soit pas toujours aussi dégradante en anglais qu'en français, puisqu'on peut être drunk as a lord ou à la rigueur as a fiddler ou as a piper, mais que, par contre, si le français ne possède guère comme comparaisons anthropomorphiques que sôkl comme un Polonais (dont certains attribuent l'origine à Napoléon lors de sa victoire du col de Somosierra) ou comme trente mille hommes, il compare volontiers l'ivrogne à toute une série d'animaux plus ou moins vils : l'âne, la bourrique, le cochon; la grive, le hanneton, la vache. En anglais, les animaux utilisés dans les comparaisons ne sont guère que la mouche, la salamandre et un seul, la truie, considéré comme répugnant (drunk as Davy's, ou David's sow). L'usage nord-américain puise évidemment pour ses comparaisons dans la faune locale : angl. : drunk as a skunk, où l'on peut noter la rime; fr. : soûl comme un ours. La remarque d'Albert Dauzat ${ }^{10}$ selon laquelle "le rava'ement le plus fréquent dans tous les argots est celui de l'homme à l'animal » se trouve donc vérifiée davantage, dans ce cas particulier, en français qu'en anglais.

\section{DIFFÉRENCES GÉOGRAPHIQUES}

Si les synonymes de drunk se sont multipliés aux États-Unis à l'époque de la prohibition, un Drinkers Dictionary ${ }^{11}$ avait déjà été pub'ié en 1737 dans deux journaux appartenant à Benjamin Franklin et les expressions qu'il contenait, comme «he's been at an Indian feast», "he makes Virginia Fence» ou «he's got the Indian vapours $\gg$ étaient d'usage strictement américain. Le dictionnaire d'argot d'Eric Partridge ${ }^{12}$ signale l'origine américaine, australienne ou néozélandaise de certains termes mentionnés.

De même en français, on pourrait faire la liste des expressions synonymes d'ivre, en usage au Canada. Ceráaines accusent l'influence de l'anglais : «être bine » ou « rond comme une bine », «frosté », «parti sur une tripe». La plupart des expressions entrent d'ailleurs dans l'une ou l'autre des catégories définies par Wentworth et Flexner : «être paqueté » ou «se paqueter la fraise 》, "sauter», 《prendre $»$ ou « revirer une brosse $»$, « être sur une brosse $»$ ou « une balloune », « être parti pour la gloire», etc.

\section{CONCLUSION}

Une comparaison générale de la langue familière et argotique en français et en anglais reste à faire et serait fascinante, nous semble-t-il, pour le linguiste et pour le traducteur, tout comme pour le sociologue qui s'intéresse aux différences de civilisations d'un peuple à l'autre. Nous n'avons voulu ici qu'en donner un exemple, emprunté à l'un des domaines les plus riches de l'argot.

Claude Romney

10. Cité par Eric Partridge, Slang To-Day and Yesterday, New York, Macmillan, $3^{e}$ édition, 1950 , p. 13

11. Cité par Cedric Larson, "The Drinkers Dictionary „, American Speech, vol. XII, n* 2, avril 1932, p. 87-92.

12. Eric Partridge, $A$ Dictionary of Slang and Unconventional English, 2 vol., Londres, Routledge and Kegan Paul, se éd., 1961, (1re éd., 1937). 


\section{OUVRAGES UTILISÉS POUR LA COMPILATION DES TERMES}

Bauche, Henri, le Langage populaire, Paris, Payot, 1920.

Chautard, Émile, la Vie étrange de l'argot, Paris, Denoël et Steele, 1931.

Farmer, J.S. et W. E. Henley, Slang and Its Analogues, 7 vol., Londres, Routledge and Kegan Paul, 1890-1904, réimpression New York, Kraus Reprint Corp., 1965.

Fowler, H.W., A Dictionary of Modern English Usage, Oxford, Oxford University Press, 1940, 1re éd., 1926.

Girard, Robert, le Royaume d'argot, Paris, Denoël, 1965.

Marcillac, Jean, Dictionnaire français-argot, Paris, Éditions de la pensée moderne, 1968.

Marks, G.A. et C.B. Johnson, Harrap's English-French Dictionary of Slang and Colloquialisms, Londres, George G. Harrap and Co., 1975.

Perreau, Robert et Margaret J. Langford, Lexique américain-français de la langue idiomatique, Paris, Éditions Ophrys, 1972.

Prenner, Manuel, * Slang Synonyms for Drunk \#, American Speech, vol. 4, n 2, đécembre 1928 , p. 102-103.

Prenner, Manuel, \&Drunk in Slang - Addenda », American Speech, vol. 16, no 1, février 1941 , p. 69-70.

Prenner, Manuel, * More Slang Words for Drunk \$, American Speech, vol. 5, no 6, août 1929 , p. 441. 